\title{
Study of object identification architectures for internet of things
}

\author{
THALLYSON P. DA SILVA MELO (IC), LUIS F. DE ÁVILA (PQ).
}

\begin{abstract}
According to the study of internet of things, perform a proof of concept of an example of architecture and associated protocols and also an application with sensors.
\end{abstract}

Key words: IoT (Internet of Things), M2M (Machine To Machine), Sensors;

\section{Introduction}

This research aims the theoretical study of the internet concept of things, object identifiers, architectures and protocols. The practical proof of concept is performed through two experiments: a simulation with OM2M service platform and a simulation with temperature and humidity sensor. OM2M is a global initiative to develop a single standard, global, for communication between machines focused on the service layer. The simulation consists in to control (turn on or turn off) a set of virtual lamps, individually or collectively, using a remote server. For this control, a set of services offered by OM2M platform is used. For the simulation with the sensor, a communication project is proposed using a simple integration of the Arduino microcontroller using loT. The main idea is to monitor and to control temperature and humidity. The application will include a database that stores the temperature and the humidity and from that information, will be presented graphics in real time.

\section{Results and Discussion}

For OM2M simulation, since the OSGi is configured and there is a connection with the NSCL, is a possible to access an online server OM2M, to perform remote management of NSCL and GSCL. The web NSCL interface is accessed according to an IP network address. The GSCL interface is accessed through the NSCL interface and with the NSCL and GSCL services running, is possible to start the control of lamps, via remote terminal, from three commands: turn the lamp 1, turn on the lamp 2 or connect the two lamps at the same time. Thus, it ends up successfully configuring the required application.

For the simulation with the sensor, the initial result is to monitor the temperature and humidity charts in IBM Quickstart, obtained by entering a MAC address contained in the application code. The final result is the relation between the NodeRed and the database DB MongoLab sandbox, creating a HTTP service that results in a base that enables query to check a graph of the parameters (temperature and humidity) from a certain time and range of parameter values, without inserting the MAC address.

\section{Conclusions}

In conclusion, through the theoretical study of the internet of things, it was possible to perform two practical simulations to identify its concept by checking the operation of a OM2M architecture, with their proper services, and analyzing the interaction between a temperature and humidity sensor with Arduino, providing results in an online HTTP service, this, based on a database and an IBM server.

\section{Acknowledgement}

The authors thanks to the Programa Institucional de Bolsas de Iniciação em Desenvolvimento Tecnológico e Inovação $\mathrm{PIBITI/CNPq-Funttel} \mathrm{for} \mathrm{the} \mathrm{financial} \mathrm{support.}$

\footnotetext{
${ }^{1}$ Yassine Banouar, Thierry Monteil, Mahdi Bem Alaya, Christophe Chassot, Khalil Drira. "OM2M: Standardized service platform for M2M interoperability". Eclipsecon France 2014.

${ }^{2}$ The eclipse Foundation. "OM2M Connecting things,

"http: / /eclipse.org /om2m /".

${ }^{3}$ Kyle Brown, "Build a cloud-ready temperature sensor with the Arduino Uno and the IBM IoT Foundation"

IBM developerWorks. ( $)$ Copyright IBM Corporation 2014. All Rights Reserved.
} 